\title{
Biomimesis: nuevos horizontes de sostenibilidad y tendencias globales de la praxis tecno-científica en el mundo contemporáneo
}

\author{
Biomimicry: new sustainability horizons \\ and global tendencies of techno-scientific \\ praxis in the contemporary world
}

\author{
Biomimética: novos horizontes de sustentabilidade \\ e as tendências globais da prática tecnocientífica \\ no mundo contemporâneo
}

\author{
Carlos Hugo Sierra Hernando \& Nelly Kuiru² \\ 'Doctor en Sociología 2Indigena Uitoto - Clan Jitomagaro (Amazonas) \\ 'Universidad del País Vasco / Euskal Herriko Unibertsitatea (UPV-EHU). \\ Barrio Sarrienla S / N 48940 Leioa (Bizkaia) País Vasco-España. \\ ${ }^{2}$ Comisión Nacional de Comunicadores de los Pueblos Indígenas de Colombia \\ 1,2carlos.ehu@gmail.com
}

\section{Resumen}

El propósito fundamental de este artículo es exponer de modo sinóptico algunas claves esenciales del enfoque biomimético, en la medida en que constituye un modo específico de concebir la naturaleza que está propiciando notables avances en numerosas disciplinas tecno-científicas de vanguardia. En este sentido, la biomimesis no sólo puede aportar claves prácticas para desarrollar modelos de una ecología optimizada y autosuficiente en los entornos humanos sino que también abre una vía reflexiva respecto a la orientación operativa y a la potencialidad de la propia tecnociencia, entendida como una metodología de investigación característica de nuestras sociedades contemporáneas, para desarrollar un horizonte de sostenibilidad global en el futuro.

Palabras clave: tecnociencia, biomimesis, desarrollo sostenible, epistemología, naturaleza.

\begin{abstract}
The fundamental propose of this article is to expose synoptically some essential keys of biomimetic approach, based on that it constitute an specific way of conceiving nature, that is promoting remarkable advances in many techno-scientific disciplines at the vanguard. In this sense, biomimicry not only can provide practical keys for developing models of an optimized and auto-efficient ecology in human surroundings but also it opens a reflexive road around the operative orientation and the potentiality of the techno-science itself, understood as a investigation methodology characteristic of our contemporary societies, for developing global sustainability horizon in a future.
\end{abstract}

Key-words: technoscience, biomimicry, sustainable development, epistemology, nature. 


\section{Resumo}

O principal objetivo deste artigo é apresentar de modo sinóptico algumas chaves essenciais da abordagem biomimético, na medida em que constitui uma forma específica de pensar sobre a natureza que está levando a progressos significativos em muitas disciplinas técnico-científicas de ponta. Neste sentido, biomimetismo não só pode fornecer pistas para desenvolver pistas práticas para desenvolver modelos de uma ecologia otimizada e autossuficiente nos entornos humanos, mas também abre uma forma cuidadosa em torno de orientação operacional e o potencial da própria tecno-ciência em si, entendida como uma metodologia o recurso de pesquisa das nossas sociedades contemporâneas para desenvolver um horizonte de sustentabilidade global no futuro.

Palavras-chave: tecno-ciência, biomimetismo, desenvolvimento sustentável, epistemologia, natureza

"El intento, intrínseco a la ciencia natural, de introducirse en el entero universo mediante un método que aísla e ilumina a un objeto tras otro, progresando así de una a otra conexión de hechos, se refleja en la técnica, en cuanto ésta, paso tras paso, se insinúa en dominios siempre nuevos, va transformando el Universo ante nuestra mirada, y le da la forma de nuestra propia imagen".

"Porque la esencia de la técnica moderna reposa en lo dispuesto, tiene que aplicar la ciencia natural exacta. De eso surge el engañoso parecer que la técnica moderna es ciencia natural aplicada. Este parecer puede mantenerse mientras no haya indagado suficientemente ni el origen esencial de la ciencia moderna, ni la esencia de la técnica moderna".

M. Heidegger. La pregunta por la técnica (Die Frage nach der Technik), 1949.

\section{Introducción}

La firme difusión del enfoque biomimético (término popularizado en los últimos años por la bióloga estadounidense Janine $M$. Benyus ${ }^{1}$ y que procede, a su vez, del binomio griego ßíos, bios -vida- $y$

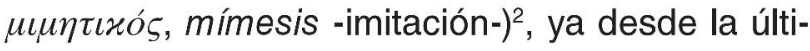
ma década del siglo XX, en el campo de la tecnociencia aplicada de vanguardia, ha supuesto, en el fondo, dotar de nuevos matices y perspectivas a la mirada escrutadora del investigador o del ingeniero contemporáneo.

El interés depositado en la naturaleza como fondo de provisión de fórmulas prácticas y eficaces a muchos de los problemas humanos hasta entonces irresueltos, acarrea una novedosa modulación en los focos de interés para el "ojo de la ciencia" (conforme a la afortunada expresión de A. L. Peck) ${ }^{3}$. Aún cuando esta idea, en realidad, se pierde en la noche de los tiempos y atraviesa los milenios de antigüedad que acompañan a las civilizaciones humanas, sólo ahora la ciencia ha reparado y sopesado con interés las consecuencias prácticas de una postura intelectiva que, dependiendo del enfoque interpretativo que se desee ejercer, alberga una enorme carga de indeterminación y ambivalencia. En cierto modo, como si se arrogara en su literalidad el antiguo mitologema jánico ${ }^{4}$, aquí la ciencia presenta también dos caras completamente contradictorias y mutuamente excluyentes (como resultado del grado de consistencia 
otorgado a la mediación tecnológica), y las implicaciones que se desprenden respecto a la toma de postura por una o por otra se manifiestan, en términos de sostenibilidad e insostenibilidad antropogénica, en el orden natural a escala planetaria.

Convendría retener este sutil planteamiento, a todas luces elocuente y esclarecedor, porque ocurre, a fin de cuentas, que se ha concedido, a nuestro entender, excesiva trascendencia a las espectaculares innovaciones tecnológicas que se han ido desarrollando mediante la emulación inquisitiva de la funcionalidad natural, mientras que se han obviado las consecuencias de fondo que el enfoque biomimético acarrea en el campo de la epistemología científica y, en última ins- tancia, en lo que concierne al modelo último de desarrollo humano. Al fin y al cabo, incluso en la ciencia contemporánea de vanguardia no es posible sustraernos a la conducción teórica como guía constitutiva de la actividad fáctica ${ }^{5}$. Es por ello que, en este caso, los árboles no nos han dejado ver el bosque y, desde este punto de vista, resulta imprescindible prestar también atención al trasfondo teórico-reflexivo de la tecnociencia y el modo en que concibe la biomímesis ya que, tal y como nos recuerda Leonardo di ser Piero da Vinci (pionero, a su vez, de la biomimética ingenieril renacentista), "los que se enamoran de la práctica sin la teoría son como los pilotos sin timón ni brújula, que nunca podrán saber a dónde van"6.

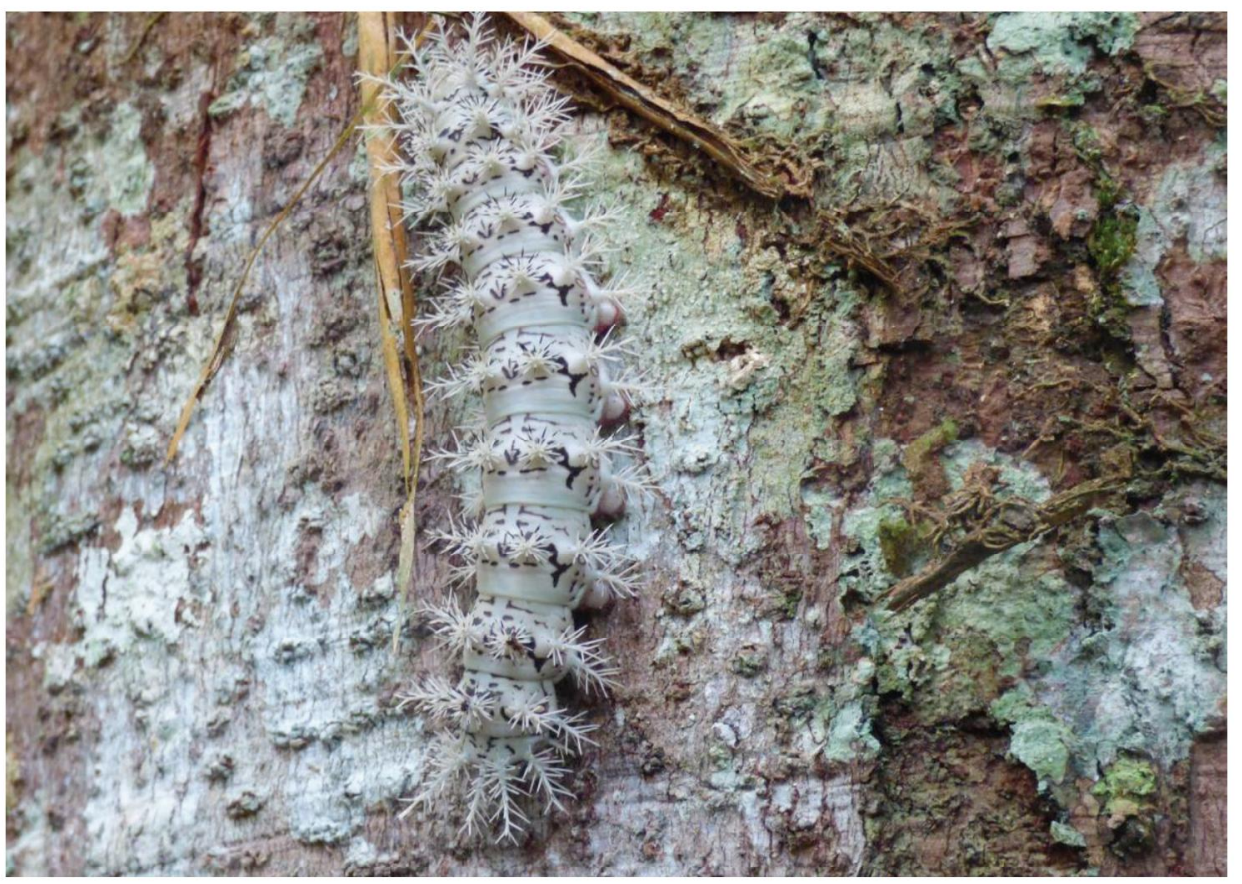

Figura 1. Mimetismo natural. Taturana Oblicua (Ionomía obliqua). Imagen: Nelly Kuiru

\section{Claves epistemológicas en la tecnociencia}

El desarrollo vertiginoso de la praxis tecnocientífica (término acuñado por el filósofo de la ciencia Gastón Bachelard en $1953^{7}$ y posteriormente difundido por Bruno Latour en los años ochenta ${ }^{8}$ ) en tanto que proceso concluyente de las tendencias de ajuste de la investigación científica que se anticipan en la llamada Big Science (Alvin M. Weinberg ${ }^{9}$ \&
Derek J. de Solla Price ${ }^{10}$ ) revela transformaciones de gran envergadura que, hasta el momento, no se han calibrado en toda su integridad.

En primer lugar, con la convergencia e inclusión a gran escala de las características (sintetizadas de modo incontrovertible por Hans Radder ${ }^{11}$ ), de 
los valores esenciales (eficacia, eficiencia, aplicabilidad, utilidad...) y de los patrones procedimentales del sistema y del fenómeno tecnológico (de acuerdo a la terminología de J. Ellul'12) en la praxis científica moderna y, más allá, en la empresa racional de modelización clásica de la realidad natural, vienen a incorporarse y a sobresalir presupuestos intra-teóricos que dan lugar a la producción de un conocimiento instrumental con fines altamente operativos ${ }^{13}$.

En realidad y salvando las distancias, esta circunstancia ya palpita de forma solapada y en menor medida en las antiguas raíces de la racionalidad occidental, lo que nos obliga a retrotraernos a los preludios incipientes de la comprensión protocientífica, donde se vislumbra un antiguo rasgo elemental de toda propensión intelectiva: la perplejidad inherente ante lo que acontece en el mundo real que circunda al ser humano, entendida como un eficaz catalizador para la producción fundamentada del conocimiento. Este asombro especulativo ${ }^{14}$, que constituye la antesala para el nacimiento y progreso de la destreza epistémica, queda difuminado históricamente, durante un paulatino y discreto proceso de inversión de valor (entre contemplación y acción) que se remonta, aunque de modo subyacente, a las poderosas abstracciones de los antiguos filósofos griegos (en especial, la escuela atomista), en cuanto se da inicio al bosquejo de una imagen controlable y predecible del mundo, hasta imponerse, en consecuencia y ya a partir de la revolución epistemológica del siglo XVII, un modelo clásico de ciencia activa y operativa. Ciertamente, la audaz aventura de ordenamiento geométrico del mundo, inaugurado hace más de dos mil años de forma

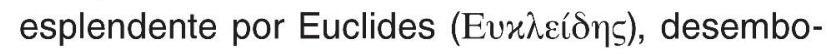
ca de manera rutilante, en la puesta en marcha del proyecto mecanicista continental de $R$. Descartes (siglo XVII) y en la revolución matemática establecida por Galileo (a partir de la cual y, de acuerdo con Alexandre Koyré, el espacio concreto de la física antigua y medieval, el cosmos en definitiva, va a ver sustituido por un universo abstracto) ${ }^{15}$, dejando el espacio expedito para el advenimiento de la mecánica de Newton y su cuadro clásico del mundo (o, lo que Milič Čapek denomina "la idea cinético-corpuscular de la naturaleza") ${ }^{16}$.

Ahora bien, en este curso histórico, sujeto, no tanto a un proceso de rectilíneo de avance acumulativo, sino más bien a cuantiosas mutaciones (en el sentido dado por $\mathrm{G}$. Bachelard ${ }^{17}$ ) y enconadas contiendas internas en pos de la supremacía heurística dentro del ámbito científico (inspirándonos en el célebre planteamiento teórico de T. Kuhn ${ }^{18}$ acerca de la inconmensurabilidad entre paradigmas y el cuestionamiento de la idea de progreso en la ciencia llevada a cabo por las corrientes epistemológicas posteriores), resulta imprescindible prestar atención al exitoso afianzamiento que, en nuestros días, ha logrado (con sus correspondientes variantes e innovaciones), aquella vertiente epistémica de largo alcance que se fundamenta en la instrumentalización inductiva y en el dominio fáctico de la naturaleza, circunstancia ésta preconizada ya por el mismísimo $\mathrm{F}$. Bacon (siglos XVI \& XVII) ${ }^{19}$.

En tal sentido, se ha de considerar el proyecto baconiano, cuyo papel, digamos de paso, en la fundación de la ciencia clásica se encuentra, sin ninguna duda, incorrectamente sobrevalorado ${ }^{20}$, como un precedente sin igual de aquella tendencia ligada a la praxis, muy cercana a la "física de la explotación de las cosas" en base a una "ciencia de ingeniero" 21 , que iba a triunfar de modo parcial en el modelo de ciencia clásica (con el descollante corolario de la llamada Revolución industrial) y ya absolutamente tras el cambio axiológico experimentado en las últimas décadas con el advenimiento de lo que se ha venido a denominar como tecnociencia. 




Figura 2. Utilización biomimética de alcaloides. Rana Dardo (Dendrobatidae). Imagen: Nelly Kuiru

Pero eso no es todo. En segundo lugar, es preciso tener en cuenta el papel de la tecnociencia como fuerza motriz del proceso de globalización contemporánea ${ }^{22}$, en tanto que, a través de sus cada vez más sofisticadas innovaciones artefactuales, estimula lógicas de mutación perceptiva de las coordenadas espacio-temporales y adquiere una inusitada preponderancia en la conformación de un nuevo escenario humano de interactividad.

Esta inédita situación de centralidad de la ciencia dentro del contexto social moderno acaece tras la progresiva adaptación de las metodologías y estrategias de gestión de la investigación a los criterios de actuación y exigencias particulares de la ciencia industrial (desde Justus von Liebig en adelante $)^{23}$ y la definitiva superación de los espacios (fundamentalmente, departamentos universitarios y laboratorios gubernamentales) donde se llevaba a cabo la actividad científica institucionalizada (sumándose a los mismos parques científicos, ciudades científicas, áreas de desarrollo, colaboratorios o laboratorios distribuidos, laboratorios vivientes, empresas de base tecnológica, spin off, etc. $)^{24}$. Nos hallamos en una nueva cultura de la investigación que enfatiza un modo de hacer ciencia que va más allá de las fronteras impuestas por la acade$\mathrm{mia}^{25}$ y que viene a finiquitar, debido a su ínsito pragmatismo, el ethos de autonomía ideal asignado al quehacer científico tal y como fue propuesto por R. K. Merton en su momento ${ }^{26}$.

No obstante, la adopción por parte de la actividad tecnocientífica de una axiología centrada en los criterios finalistas y utilitarios del sistema de producción e innovación contemporáneos conlleva, por un lado, una descontextualización de su metodología exploratoria (Hugh Lacey ${ }^{27}$ ) frente a la ciencia clásica, $y$, por otro, una honda reformulación de su universo de valor y de su capacidad de maniobrabilidad y legitimación en términos éticos en relación con todas aquellas problemáticas humanas en las que, de modo directo o indirecto, lleva a cabo una función protagónica. No es casual, en definitiva, que la orientación con la que avanza la tecnociencia moderna haya entrado en seria consideración como objeto controvertido de debate en lo que tiene que ver con las amenazas de índole ambiental que asolan los ecosistemas a escala planetaria. 


\section{Tecno-ciencia \& Biomimesis}

Este modo concreto de resolver el problema de la legibilidad significativa del mundo (que evoca, tal y como sostiene $\mathrm{H}$. Blumenberg, una experiencia contingente de lo natural como mera disponibili$\left.\operatorname{dad}^{28}\right)$, ha extendido por doquier la presunción, sustentada en un convincente y elemental optimismo racionalista, de una mejora incesante $y$ segura de las condiciones de auto-organización social a través del potencial de perfectibilidad que atesora el desarrollo tecno-científico y, por ende, a la elusión del orden natural como un factor determinante en la evolución, presente y futura, de las capacidades civilizatorias de la vida humana.

En este sentido, la crítica contemporánea a esta perspectiva, en la que subyace un programa doctrinario de antropocentrismo extremo que acrecienta el cisma entre la tecnosfera y la biosfera (tal y como subraya Andrew Dobson, quien distingue entre aquella postura que exalta la supremacía instrumental sobre el mundo y la perspectiva protagórica, más ponderada, sujeta a una gnoseología cuyo valor se fundamenta en el inherente relativismo de la perspectiva humana ${ }^{29}$ ), pasa por desechar y reorientar las bases socioeconómicas del proyecto actual de desarrollo humano a escala planetaria, lo que por lógica debe entrañar, al fin y al cabo, la necesidad de reintroducir la naturaleza como una variable esencial de cálculo a la hora de calibrar el alcance e impacto de las externalidades antropogénicas, positivas y negativas, que se producen en todos los planos de la vida terrestre. Nada más cierto a este respecto que lo dicho por el filósofo y antropólogo francés Paul Ricoeur cuando nos recuerda, casi como una exhortación admonitoria, que "formamos parte de la Naturaleza; hay que volver a situar al ser humano dentro de los ecosistemas. Somos un fragmento de la biosfera, pero el único que está dotado de conocimiento y responsabilidad. Es necesario por ello equilibrar el sentimiento de pertenencia a la naturaleza con el sentimiento de excepcionalidad del ser humano dentro de la naturaleza. Se trata de un equilibrio que hay que reconstruir continuamente"30.
Ahora bien, la puesta de relevancia del enfoque biomimético, en tanto que disciplina desde la que cabe auspiciar, al menos en apariencia, la valoración de los ecosistemas naturales, no ya como simples reservas extractivas y de suministro, sino, más bien, como modelos referenciales de actividad e interacción metabólica circular y auto-sostenible susceptibles de ser aplicados y transferidos a diferentes contextos sociales, trae como consecuencia lógica el repensamiento profundo del sistema internalista de producción y organización de la praxis tecno-científica contemporánea (que, digamos de paso, apenas conserva puntos de conexión con el modus operandi de la ciencia clásica ${ }^{31}$ ), así como el propio cuadro axiológico y teleológico a través de la cual orienta su actividad, sin olvidar tampoco la visión general de la realidad natural que de ella se desprende. De no ser así, subsiste el riesgo incuestionable de que el concepto de biomimesis acabe siendo fagocitado dentro de las redes operativas de producción del conocimiento científico y que su sistema de referencia representacional (haciendo nuestro el planteamiento de la teoría del Actor-Red, ANT ${ }^{32}$ ) o su trasfondo semiótico pueda verse transformado de acuerdo a criterios estratégicos (como así ha ocurrido con el término de "desarrollo sostenible", circunstancia que', en los últimos años, ha sido puesta en evidencia desde distintas teorías críticas ligadas, especialmente, al ecosocialismo ${ }^{33}$ y al enfoque decrecionista ${ }^{34}$ ), hasta el extremo de convertirse en un concepto clave que legitime el mero acercamiento al entorno natural como un acto que refrenda moral y funcionalmente el aprovechamiento utilitarista del mundo natural.

Es por ello que, a nuestro entender, los debates reflexivos en torno a la trascendencia y alcance de la acción biomimética (en su significación tradicional relacionada con la imitación de la naturaleza a la hora de reconstruir los sistemas productivos humanos, con el fin de hacerlos compatibles con la biosfera) no deben limitarse solamente al ámbito de la filosofía ecológica o de la filosofía económica crítica, sino que resulta imprescindible analizar los cambios retórico-ideológicos en el plano 
metadiscursivo (en términos de una lectura foucaultiana $^{35}$ ) y las consecuencias que acarrea todo ello en el ámbito de la epistemología (teniendo como punto de referencia el Programa
Fuerte de la Escuela de Edimburgo y sus derivas posteriores en el campo de la sociología del conocimiento científico ${ }^{36}$ ).



Figura 3. Mimetismo natural. Víbora Pudridora (Bothrops asper). Imagen: Nelly Kuiru

Desde este punto de vista, la biomimesis, entendida como una noción base desde que la que se incentiva la coevolución ajustada de los sistemas humanos y los sistemas naturales (de acuerdo con la perspectiva desarrollada por Richard B. Norgaard ${ }^{37}$ ), permite ampliar el debate en torno a las visiones generales y los fines últimos que guían la praxis tecnocientífica, más allá de las restricciones impuestas por parte de la biomimética o biognosis ingenierilis.

No se trata de una cuestión baladí. En la medida en que la tecno-ciencia se ha convertido en los últimos decenios en el impulsor y agente de globalización más importante (no hay más que revisar, al respecto, los datos del UNESCO Science Report, 2010), el desatender todo lo que concierne a este asunto supondría no abordar en toda su complejidad el problema contemporáneo en torno al desarrollo sostenible. Por lo tanto, uno de los problemas esenciales que se suscitan en torno al principio biomimético es que la inspiración y la búsqueda de la viabilidad funcional en el terreno de los procesos naturales no llegue a estimular, de modo paralelo, una reorientación de calado en los esquemas operacionales y finalistas de la praxis tecno-científica, sino que más bien, suponga ahondar en la consideración de la biodiversidad como un inmenso reservorio de propiedades, características y funciones sujeto a una lógica de intervención puramente instrumental.

\section{Biomímesis: más allá de una ingeniería inversa sobre la naturaleza}

Si se presta atención al paradigma biomimético que ha imperado, desde el inicio de la década de los noventa hasta nuestros días, en determinadas disciplinas y áreas del conocimiento vinculadas a la investigación aplicada que se han demostrado claves en la acelerada propagación de la tecnociencia contemporánea (por ejemplo, la ciencia de los biomateriales, la nanotecnología, la biotecnología, la farmacología, la óptica, la arquitectura, 
la robótica, la bioingeniería, etc.), cabe llegar a la conclusión de que, en realidad, no ha traído como consecuencia un cambio substancial en las concepciones de fondo, en las lógicas de transferencia de conocimiento o en los procedimientos operativos que caracterizan al desarrollo tecnológico moderno.

Desde este punto de vista, la introducción de la perspectiva biomimética en los modos de experimentación, innovación e, incluso, explotación comercial por parte de laboratorios de vanguardia, institutos de investigación o empresas de alta tecnología, no ha supuesto, aunque, en efecto, difiera de la bio-utilización o de las tecnologías de bioasistencia, la apertura de líneas de reflexión novedosas o de gran alcance dentro de las corrientes establecidas en los estudios STS (Science and Technology Studies) ${ }^{39}$. Y la razón de ello es que, en el fondo, ha imperado la tendencia a explorar los procesos de funcionalidad natural como fuente de inspiración en el diseño de invenciones que se revelan mucho más eficaces para la resolución de problemas prácticos en el contexto de las sociedades humanas, pero, eso sí, bajo unas coordenadas específicas de interacción gnoseológica entre los equipos de innovación y desarrollo tecnológico y el propio entorno natural (ya sea desde el diseño a la biología o viceversa) que se asemejan más a lo que se podría reconocer como una especie de "ingeniería inversa" (es decir, la mera obtención de información o un diseño útil a partir de los modelos biológicos de la naturaleza, con el fin de determinar de qué está hecho, qué lo hace funcionar y cómo se alcanzó tal o cual propiedad en el estadio evolutivo).

La naturaleza se convierte, de este modo, en un gigantesco y casi inagotable "banco de ideas" no sometido a las restricciones de las leyes internacionales de propiedad intelectual, cuya fértil potencialidad acaba siendo materializada en un sistema procedimental de diseño e innovación para ser introducida, posteriormente, dentro de la lógica competitiva del mercado.



Figura 4. Aplicación biomimética de la aerodinámica de los odonatos. Libélula amazónica. Imagen: Nelly Kuiru 
Desde esta perspectiva, a todas luces marcadamente reduccionista, la puesta de relevancia del concepto biomimesis en la literatura científica ha conseguido incrementar el propio valor de la naturaleza, no tanto como un bien en sí mismo que asegura la vida sobre el planeta (bajo la óptica, más allá del naturalismo acrítico, de una sofisticada ética sensocéntrica y biocéntrica ${ }^{40}$ ), sino como expresión de una inteligencia evolutiva que permite contribuir, bajo una óptica de rentabilidad económica, a la resolución de los problemas fácticos en el sistema humano. No hay más que señalar algunos ejemplos de gran representatividad, a cada cual más espectacular, en los que se aplica los modelos de inspección y diseño biomimético, para evidenciar tal circunstancia.

De este modo, y dentro del área amazónica, cabe citar los trabajos de la Universidad Internacional de Florida (a través de su departamento de ciencias biológicas) en torno a la aplicación de los sistemas de protección contra el exceso de irradiación lumínica desarrollados, a través del componente de iridiscencia azul de sus hojas, por ciertas plantas del sotobosque sombrío amazónico, a ciertos sistemas de protección de reactores químicos o superficies fotosensibles ${ }^{41}$; por otra parte, el Departamento de Ingeniería mecánica y aeroespacial de la Universidad de California (San Diego) estudia la conformación física y la reducida masa del pico del Tucán (Toco tucan), compuesto por una espuma y membranas de proteína en el interior y por capas hexagonales de queratina en la parte exterior, con el objeto de explorar la posibilidad de desarrollar sofisticados componentes para aviones ultraligeros ${ }^{42}$; en dicho Departamento de la Universidad de California (dentro del programa de Ciencia de los Materiales) estudian también las escamas del Pirarucu (Arapaima gigas), compuestas internamente de fibras de colágeno y externamente de un revestimiento altamente mineralizado, con el fin de producir materiales compuestos flexibles que se combinen con superficies cerámicas duras ${ }^{43}$; al mismo tiempo, en ciertas corrientes y equipos de trabajo en el campo de la arquitectura se estudia la capacidad de las hormigas (Mycocepurus goeldii) para evitar inundaciones (mediante el cultivo de hongos) y generar microclimas a través de construcciones en forma de árbol con la idea aplicar dichas propiedades al diseño de casas subterráneas ${ }^{44}$; en el Laboratorio de Física del Estado Sólido de la Universidad de Namur se investiga el exoesqueleto del escarabajo Hércules (Dynastes hercules), y su propiedad de modificar su color en relación con el grado de humedad existente, para implementar sensores de humedad; así mismo, en varios centros de óptica se investiga el sistema ocular de las pirañas (Pygocentrus), cuya capacidad de captar la longitud de onda correspondiente al rojo le permite ver en las aguas oscuras del Amazonas, para aplicarlo a sistemas de navegación en alta mar y submarina ${ }^{45}$. No nos vamos a extender más porque la lista de ejemplos, ciertamente, llega a ser interminable.

Ahora bien, convendría tener en cuenta que este nuevo punto de referencia en lo que tienen que ver con los procesos de interpretación de los ciclos, dinámicas y propiedades funcionales de los sistemas naturales y con los propios modelos de adaptación iterativa con el entorno ecosistémico puestos en marcha por la pujante tecno-ciencia contemporánea no supone necesariamente una reorientación internalista de su praxis transformadora a escala planetaria en base al principio de precaución (ya que difiere y va más allá del mero cumplimiento de los criterios de actuación bajo el enfoque de la ingeniería biomimética) ${ }^{46}$ y al principio del límite (de acuerdo a la filosofía de la responsabilidad desarrollada por Hans Jonas) ${ }^{47}$. La demostración más evidente de que lo que se afirma aquí posee trazas de verosimilitud es la creciente convicción en determinados círculos científicos, aunque por ahora únicamente en el plano de la especulación teórica, de la factibilidad tecnológica, cada vez más cercana en el tiempo, de sortear la escasez drástica de recursos naturales terrestres mediante la terraformación ${ }^{48}$, esto es, la creación de biosferas en otros hábitats planetarios 0 , simplemente, la explotación de recursos mediante la colonización de otros planetas. 


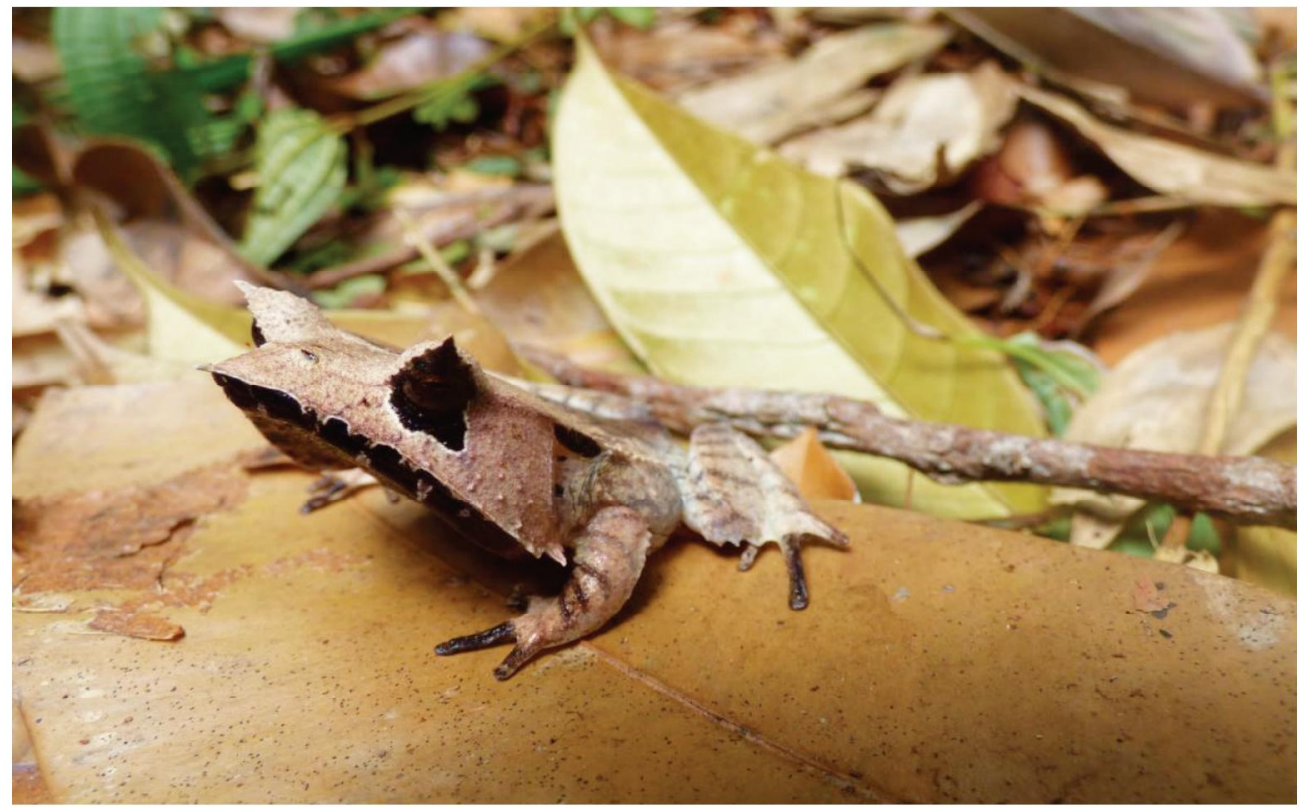

Figura 5. Mimetismo natural. Rana Cornuda (Ceratophrys cornuta). Imagen: Nelly Kuiru

En este sentido y como respuesta de lo anterior, han aparecido en escena diversos autores, como los ya citados Janine M. Benyus o Jorge Riechmann, que defienden la necesidad de una interpretación más profunda y de mayor alcance para el concepto de biomímesis. Lejos de limitarse a corroborar la validez práctica de la ingenierización de la biología a nivel de los organismos aislados, obviando de esta forma el contexto vital en el que éstos se desarrollan, se considera más adecuado vindicar un enfoque global, que preste atención, sobre todo, a la lógica innata de interdependencia desde la que emergen y conservan los ecosistemas naturales. Precisamente y con la vista puesta en este aspecto, Janine M. Benyus llega a identificar y señalar 10 propiedades esenciales privativas de los sistemas naturales:

1. Funcionan a partir de la luz solar

2. Usan solamente la energía imprescindible

3. Adecúan forma y función

4. Lo reciclan todo

5. Recompensan la cooperación

6. Acumulan diversidad

7. Contrarrestan los excesos desde el interior

8. Utilizan la fuerza de los límites

9. Aprenden de su contexto

10. Cuidan de las generaciones futuras
A decir verdad, el impacto teórico de lo expresado por J. M. Benyus resulta ilustrativo para comprender que, hasta cierto punto, nos hallamos ante una encrucijada en lo que atañe a las tendencias futuras de la racionalidad tecno-científica. Quien analice con cierto detalle ( $\mathrm{y}$ alejado de toda pretensión tecnofóbica) las implicaciones resultantes del empleo en el discurso científico de la noción de biomímesis reconocerá sin dificultad un disyuntiva de gran calado: o bien se recurre de modo particular a las resoluciones operativas concretas que se reconocen en el entorno natural con el claro propósito de paliar instrumentalmente las externalidades negativas (causadas por la gestión lineal e insostenible de los flujos de materia y energía) que acompañan a la economía capitalista industrial 0 , por el contrario, se favorece una perspectiva más incluyente y general, desde la que se posibilite reformar los sistemas organizativos que vertebran el espacio socioesférico y tecnosférico hasta lograr consolidar un patrón metabólico de intercambio material circular, absolutamente compatible y coactuante con la biosfera. 


\section{Conclusión}

A tenor de todo lo expuesto con anterioridad, cabe afirmar que la prospección del paradigma biomimético posee la virtualidad de resituarnos en el centro de la recurrente controversia acerca la supuesta neutralidad de valores -Wertfreiheit- en la estructura proposicional "objetiva" consensuada por la comunidad científica (puesta sobre la mesa de modo emblemático por M. Weber en $1919^{49}$ ) $y$ en las directrices finalistas del avance tecnocientífico teniendo en cuenta sus impactos sobre el medio ambiente a escala mundial. La biomímesis puede servir, además, de referente universal normativo y compensatorio frente a la incuestionable ausencia de una "visión o sentido del mundo" que rija la actividad de la tecno-ciencia contemporánea, considerada como un sistema de conocimiento organizado sobre la base de un alto nivel de especialización. Pero tampoco debe pasar inadvertido que el enfoque biomimético refuerza el giro descriptivo de la existencia como una realidad compleja e implicada (siguiendo a David Bohm ${ }^{50}$ ). Detrás del principio biomimético de interdependencia co-evolutiva de toda interacción vital palpita una idea de organización compleja que viene a afectar, no sólo a los organismos biológicos, sino también a los sistemas sociales modernos, espacios autorreferenciales ("autopoiéticos" diría Niklas Luhmann, trasladando el neologismo propuesto por H. Maturana y F. Varela al medio social ${ }^{51}$ ) que se regulan y acoplan estructuralmente mediante la permeabilidad selectiva de los influjos y relaciones con el medio ambiente. Se trata, en definitiva, de considerar la biomímesis como punta de lanza de un proyecto de desarrollo humano que se fundamente en la certidumbre elemental de que tanto el espacio social como el natural constituyen, a fin de cuentas, sistemas sinergéticos y cooperativos.

\section{Fotografías}

Área selvática (Leticia, Amazonas, Colombia)

\section{Literatura citada}

1. Benyus, J. M. (2012). Biomímesis. Cómo la ciencia innova inspirándose en la naturaleza. Barcelona: Tusquets Editores.

2. Benyus, J. M. \& Lehn, J-M. (2012). Bioinspiration and Biomimicry in Chemistry. Reverse-Engineering Nature. New jersey: John Wiley \& Sons, Inc., Publication.

3. C̆apek, M. (1965). El impacto filosófico de la física contemporánea. Madrid: Tecnos.

4. Dobson, A. (1997). Pensamiento político verde: una nueva ideología para el siglo XXI. Barcelona: Paidós.

5. Dryzek, John S., David Schlosberg, \& Richard B. Norgaard. (eds). (2011). The Oxford Handbook of Climate Change and Society. Oxford University Press. Oxford.

6. Hargroves, K. D. \& Smith, M. H. (2006). 'Innovation inspired by nature Biomimicry'. Ecos, (129), 27-28.

7. Jonas, H. (1995). El principio de responsabilidad: ensayo de una ética para la civilización tecnológica. BarceIona: Editorial Herder.

8. Jonas, H. (1997). Técnica, medicina y ética: sobre la práctica del principio de responsabilidad. Barcelona: Ediciones Paidós Ibérica.

9. Koyré, A. (2001). Estudios Galileanos. México, D. F.: Siglo XXI editores.

10. Koyré, A. (1979). Del mundo cerrado al universo infinito. Madrid: Siglo XXI editores.

11. Lakhtakia, A. \& Martin-Palma, R. J. (2013). Engineered Biomimicry. Amsterdam: Elsevier.

12. Lee, D. \& Thompson, M. (2011). Biomimicry: Inventions Inspired by Nature. New York: Kids Can Press.

13. Norgaard, R. B. (1994). Development Betrayed: The End of Progress and a Coevolutionary Revisioning of the Future. London and New York. Routledge.

14. Passino, K. M. (2004). Biomimicry for Optimization, Control, and Automation. New York: Springer-Verlag.

15. Riechmann, J. (2006). Biomímesis. Ensayos sobre imitación de la naturaleza, ecosocialismo y autocontención. Madrid: Catarata.

16. Riechmann, J. (2003). Todos los animales somos hermanos. Granada: Universidad de Granada.

17. Riechmann, J. (2000). Un mundo vulnerable. Madrid: La Catarata.

18. Riechmann, J. (2004). Gente que no quiere viajar a Marte. Madrid: La Catarata.

19. Shuker, KPN. (2001). The Hidden Powers of Animals: Uncovering the Secrets of Nature. London: Marshall Editions Ltd. 


\section{NOTAS}

1 Se considera que Janine M. Benyus (1958-...), cuya obra se ha convertido en una referencia de consulta esencial a este respecto, es la divulgadora más reconocida en la actualidad de aquella disposición del ser humano, que se pierde en la noche de los tiempos, hacia la especulación o la indagación productiva, en base al aprendizaje y la emulación de la "sabiduría" atesorada por la naturaleza. De acuerdo con esta autora la biomímesis se funda en tres principios básicos:

1. La naturaleza como modelo. La biomímesis es una nueva ciencia que estudia los modelos de la naturaleza para imitar o inspirarse en los diseños o procesos biológicos para resolver problemas humanos.

2. La naturaleza como medida. La biomímesis se vale de un estándar ecológico para juzgar la 'corrección' de nuestras innovaciones. Después de 3.800 millones de años de evolución, la naturaleza ha descubierto lo que funciona, lo que es apropiado y lo que perdura.

3. La naturaleza como mentor. La biomímesis es una nueva manera de contemplar y valorar la naturaleza. Inicia una era basada no en lo que podemos extraer del mundo natural, sino en lo que éste puede enseñarnos."

Benyus, J. M. Biomímesis. Cómo la ciencia innova inspirándose en la naturaleza. Barcelona: Tusquets Editores, 2012, p. 13.

2 No entraremos en este texto a dirimir la arcaica y enraizada distinción predominante en la cultura griega de los términos bíos (ßíos) y zoé (Zớ), que, de modo tan valioso, han puesto de relevancia destacados filósofos e historiadores de las ideas como, por ejemplo, Giorgio Agamben, Michel Foucault, entre otros.

3 Citado en Kuriyama, S., The expressiveness of the body and the divergence of Greek and Chinese medicine. New York: Zone Books, 1999, p. 128.

4 Nos referimos, claro está, a la deidad romana representada con dos rostros unidos por la línea de la oreja y la mandíbula, mirando en direcciones contrapuestas, ya que se le había otorgado la capacidad de conocer el pasado y el futuro, de abrir y cerrar a voluntad todo lo que se hallaba en la Tierra, de dar comienzo y fin a todo lo existente.

5 Coincidimos absolutamente con Alexandre Koyré cuando afirma que "...la ciencia, la de nuestra época, como la de los griegos, es esencialmente theoria, búsqueda de la verdad y que por esto tiene, y siempre ha tenido, una vida propia, una historia inmanente y que sólo en función de sus propios problemas, de su propia historia, puede ser comprendida por sus historiadores". Véase en Estudios de historia del pensamiento científico. Madrid: Siglo XXI, p. 385.

6 Esta cita se encuentra contenida en su Trattato della pittura, conjunto de manuscritos reunidos por Francesco Melzi en torno a 1542 y publicados por vez primera por Raffaelo du Fresne en 1651 .

7 Bachelard, G. La materialisme ratione/, Paris: PUF, 1953.

8 Latour, B. La Science en action. Paris: La Découverte, 1989.

9 Weinberg, Alvin M. (21 July 1961). "Impact of Large-Scale Science on the United States." Science 134 (3473): 161-164.

10 Solla Price, D. J (1963). Little Science, Big Science. New York: Columbia University Press.
11 Realizabilidad, sistematicidad, heterogeneidad, relación con la ciencia, división del trabajo. Véase al respecto, Radder, $H$. The material realization of science. From Habermas to experimentation and referential realism. Dordrecht: Springer, 2012; Radder, H. Nordmann, A. \& Schiemann, G. (Eds.). Science transformed? Debating claims of an epochal break. Pittsburgh: University of Pittsburgh Press, 2011; Radder, H. The World Observed / The World Conceived. Pittsburgh: University of Pittsburgh Press, 2006.

12 El fenómeno tecnológico, propio de la modernidad, transforma todas las actividades, vinculándolas enteramente a la técnica de la industria, y lo convierte en un proceso ilimitado. Véase al respecto, Ellul, J. (1989). Le bluf technologique. París: Hachette; Ellul, J. (1977). Le systéme technicien. París: Calmann-Levy.

13 De ahí podría inferirse la pertinencia de un análisis en profundidad sobre la naturaleza de la tecnología, aspecto que, por lo demás, no se va a ser tratado en este artículo, en base a las diferentes líneas heurísticas dominantes dentro de la sociología de la tecnología: enfoque de sistemas (Thomas Hughes), enfoque constructivista social (Wiebe Bijker \& Trevor Pinch) y el enfoque de actor-red (Michel Callón \& Bruno Latour).

14 En relación con ello, tan sólo señalar (ya que no es objeto central en este ensayo) la existencia en el imaginario occidental de una fecunda tradición, cuya pujanza llega hasta nuestros días (desde Aristóteles a M. Heidegger, desde I. Newton hasta A. Einstein), que entrelaza este principio actitudinal radical frente al mundo y el acto teórico de la filosofía natural o de la ciencia misma.

15 Véanse al respecto dos clásicos del autor: Koyré, A. Estudios Galileanos. México, D. F.: Siglo XXI editores, 2001.

Koyré, A. Del mundo cerrado al universo infinito. Madrid: Siglo XXI editores, 1979.

16 No referimos, claro está a Čapek, M. El impacto filosófico de la física contemporánea. Madrid: Tecnos, 1965. A este respecto, resulta también de interés Cohen. I. B. The Newtonian Revolution. Cambridge: Cambridge University Press, 1981.

17 Bachelard, G. La formación del espiritu científico: contribución a un psicoanálisis de conocimiento objetivo. México D. F.: Siglo XXI, 2007.

18 Kuhn, T. S. La Estructura de las revoluciones científicas. Madrid: Fondo de Cultura Económica, 2006.

19 A través de dos de sus obras clásicas, a saber, Novum Organum Scientiarum (1620) y Nova Atlantis (1627).

20 "Lo de "Bacon, iniciador de la ciencia moderna" es una broma, de muy mal gusto, que todavía repiten los manuales. En realidad, Bacon no comprendió nunca nada de la ciencia".

Koyré, A. Estudios Galileanos. México, D. F.: Siglo XXI editores, 2001, p. 2.

21 Citado en Koyré, A. Estudios Galileanos. México, D. F.: Siglo XXI editores, 2001, p. 2. 
22 Para conocer en profundidad el fenómeno de la globalización, sin duda hay que remitirse al filósofo alemán Peter Sloterdijk y su monumental trilogía sobre la esfericidad.

23 Habrá que recordar en este punto que la ciencia industrial comenzó a desarrollarse con vigor en Alemania durante el último tercio del siglo XIX a partir de las aplicaciones derivadas del desarrollo del electromagnetismo, la industria de los tintes basada en la química orgánica, los motores de combustión interna en base a los principios de la termodinámica, etc. Por su parte y a comienzos del siglo XX, empresas de EE.UU. como la General Electric y la American Telephone and Telegraph (ATT) transformaron sus pequeños laboratorios para trabajos rutinarios en auténticos centros de I+D+I.

24 La ciencia se convierte en eje vertebrador de un espacio dinámico de innovación y trasferencia tecnológica entre la universidad, la empresa y el gobierno bajo un modelo que Henry Etzkowitz y Loet Leydesdorff denominan de Triple Hélice (en la actualidad ya se está hablando de una Cuádruple Helice en el que se incluye el papel creciente del usuario - T. Liljemark; R. M. Yawson; J. Eriksson; T. Hämäläinen-). Véase al respecto, Etzkowitz, H. \& Leydesdorff, L. "A Triple Helix of University-Industry-Government Relations", Minerva 36 (1998) 203-288.

25 De hecho, se habla ya del advenimiento de una ciencia Modo 2 (Michael Gibbons, Camille Limoges, Helga Nowotny, Simon Schwartzman, Peter Scott \& Martin Trow), de una Ciencia Postacadémica (John Ziman) o de una Ciencia Postnormal (Silvio Funtowicz \& Jerome Ravetz).

26 Centrado en cuatro criterios básicos: Universalismo (las pretensiones de verdad se someten a criterios impersonales preestablecidos); Comunismo (los logros de la ciencia son propiedad común); Desinterés (Ios científicos no han de perseguir en sus investigaciones fines personales); Escepticismo institucionalizado u organizado (los resultados de la ciencia se consideran siempre revisables en función del desarrollo de la misma).

27 Lacey, H. Reflections on science and technoscience. Scientiæ Studia, São Paulo, v. 10, pp. 103-28, 2012.

28 Blumenberg, H. La legibilidad del mundo. Barcelona: Ediciones Paidós, 2000, p. 13.

29 Nos referimos, claro está, a la división que establece entre human-instrumental anthropocentrism y human-centred anthropocentrism, la cual hace referencia al ya clásico principio del famoso sofista griego, Protágoras de Abdera

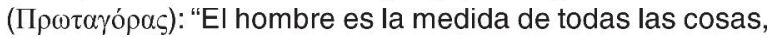
de las que son en cuanto que son, de las que no son en

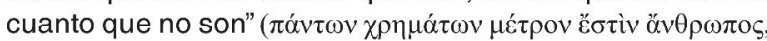

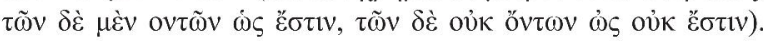
Véase al respecto: Dobson, A. Pensamiento político verde: una nueva ideología para el siglo XXI. Barcelona: Paidós, 1997.

30 Ricoeur, P. 'L'Éthique, le politique, l'écologie'. Écologie Politique, $\mathrm{n}^{\circ}$ 7, 1993, p. 14.

31 Ciertamente, la praxis científica, durante el siglo XX, ha experimentado dos transformaciones de gran calado: a partir de la segunda guerra mundial, con la propagación de la llamada "Big Science" (remitimos a los artículos clásicos de
Alvin M. Weinberg -'Impact of Large Scale Science on the United States.' Science, vol. 134, no 3473, 1961- y de Derek John de Solla Price -Little Science, Big Science and Beyond. New York: Columbia University Press, 1986-) y, en las últimas décadas, con el advenimiento de la denominada "Tecno-Ciencia" (término ideado por Bruno Latour en 1983, en su obra referencial Ciencia en acción. Cómo seguir a los científicos e ingenieros a través de la sociedad ¿Quién hace la Ciencia realmente? Barcelona: Labor, 1992).

32 En referencia a esta interesante teoría, remitimos a la obra de Bruno Latour, Michel Callon, Geoffrey C. Bowker o Susan Leigh Star.

33 Además de a Jorge Riechmann, en esta corriente podemos destacar autores como David Pepper, Michael Löwy, Joel Kovel, David Keith Orton, Ramachandra Guha, Barry Commoner, entre otros.

34 En lo que tiene que ver con este enfoque, resulta preciso consultar la obra de Nicholas Georgescu-Roegen, Herman Daly, François Schneider y, por supuesto, de Serge Latouche, tal vez el más conocido de todos.

35 Tal vez sea el historiador de las ideas y filósofo francés Michel Foucault (1926-1984) quien de forma más brillante y profunda ha puesto de relevancia la convergencia entre discurso y poder. Especialmente significativo y altamente recomendable resulta su discurso inaugural en el Collège de France, L'ordre du discours (El orden del discurso), allá por el año 1970.

36 EL programa empírico de relativismo (programa EPOR, desarrollado por H. Collins y T. Pinch), los Estudios de Laboratorio (B. Latour, S. Woolger y K. Knorr-Cetina), el programa SCOST (Social Construction of Science and Techonology: W. Bijker \& T. Pinch) o la ya mencionada teoría de Actor- Red.

37 Resulta de interés en este punto consultar los siguientes artículos de este autor: Norgaard, R. B. \& Kallis, G. 'Coevolutionary ecological economics', Ecological Economics, 69.4 (2010): 690-699; Norgaard, R. B. \& Gual, M. A. 'Bridging ecological and social systems coevolution: A review and proposal', Ecological economics, 69.4 (2010): 707-717.

38 Término citado en Riechmann, J. Biomímesis. Ensayos sobre imitación de la naturaleza, ecosocialismo y autocontención. Madrid: Catarata, 2006, p. 189.

39 Aquí entran en escena autores de gran repercusión en el estudio de la ciencia y tecnología como Carl Mitcham, Langdon Winner, Stephen H. Cutcliffe o Paul Durbin.

40 Véase al respecto, la obra de Peter Singer, Fritz Jahr, Tom Regan, Angelika Krebs, Paul Taylor, Leena Vikka o Nicholas Agar, entre otros.

41 Lee, D; Kelley, J \& Richards, J. H. 'Blue Leaf Iridescence as a By-product of Photoprotection, Tropical Rainforest Understory Plants. Botanical Society of America, 2008.

42 'Science Concentrates: Secrets of toucan beak revealed'. Chem. Eng. News. 83(50), 2005.

43 Meyer M. A; Lin YS; Olevsky EA \& Chen P-Y. 'Battle in the Amazon: Arapaima versus Piranha', Advanced Engineering Materials, 14: B1-B10, 2012. 
44 Rabeling, C.; Verhaagh, M. \& Engels, W. 'Comparative study of nest architecture and colony structure of the fungusgrowing ants, Mycocepurus goeldii and M-smithii'. Journal of Insect Science. 7(40): 1-13, 2007.

45 Shuker, KPN. The Hidden Powers of Animals: Uncovering the Secrets of Nature. London: Marshall Editions Ltd, 2001.

46 El «principio de precaución», concepto procedente del vocablo alemán "Vorsorgeprinzip", promueve el control y la reducción anticipativa de los posibles riesgos o efectos negativos para la salud pública y el medio ambiente de una actividad tecno-científica con un alto grado de incertidumbre en los protocolos de evaluación de resultados.

( kantiano, «actúa de tal manera que los efectos de tu acción sean compatibles con la permanencia de la vida humana auténtica sobre la Tierra», constituye una apelación a construir un modelo específico de progreso que tenga en cuenta la limitación de recursos, lo que impele a economizar (gestión ecológicamente viable de recursos locales) y a mantener dicho compromiso a lo largo del tiempo (distribución intergeneracional de recursos escasos y no renovables). Véase al respecto, Jonas, H. El principio de responsabilidad: ensayo de una ética para la civilización tecnológica. Barcelona: Editorial I lerder, 1995; Jonas, II. Técnica, medicina y ética: sobre la práctica del principio de responsabilidad. Barcelona: Ediciones Paidós Ibérica, 1997.
48 Dentro de este asunto, relacionado con lo que se denominó en su momento como ingeniería planetaria (Carl Sagan) o modelación planetaria (Joel Levin) se producen los mismos problemas correspondientes a la conformación de un modelo medioambiental que sea refractario al antropocentrismo radical y tendente a constituir una ética cosmocéntrica. Recordemos al respecto de la explotación de los recursos naturales de la Luna y de otros cuerpos celestes el "Tratado sobre los principios que deben regir las actividades de los Estados en la exploración y utilización del espacio ultraterrestre, incluso la Luna y otros cuerpos celestes", resolución 2222 (XVI) de 1967 promulgado por la ONU.

49 Wissenschaft als Beruf, München und Leipzig 1919, GAW 582-613.

50 Véase al respecto, Bohm, D. Wholeness and the Implicate Order. London: Routledge, 1980.

51 Para una visión sinóptica del concepto, véase Luhmann, $\mathrm{N}$. Sistemas sociales. Barcelona: Anthropos, 1998; Maturana, Humberto R. De Máquinas y Seres Vivos, autopoiesis de la organización de lo vivo. Santiago de Chile: Editorial Universitaria, 1997. 